\title{
Post-Newtonian approximation in Maxwell-like form
}

\begin{abstract}
Jeffrey D. Kaplan, David A. Nichols, and Kip S. Thorne
Theoretical Astrophysics, California Institute of Technology, Pasadena, California 91125, USA

(Received 18 August 2008; revised manuscript received 25 February 2009; published 10 December 2009)

The equations of the linearized first post-Newtonian approximation to general relativity are often written in "gravitoelectromagnetic" Maxwell-like form, since that facilitates physical intuition. Damour, Soffel, and $\mathrm{Xu}$ (DSX) (as a side issue in their complex but elegant papers on relativistic celestial mechanics) have expressed the first post-Newtonian approximation, including all nonlinearities, in Maxwell-like form. This paper summarizes that DSX Maxwell-like formalism (which is not easily extracted from their celestial mechanics papers), and then extends it to include the post-Newtonian (Landau-Lifshitz-based) gravitational momentum density, momentum flux (i.e. gravitational stress tensor), and law of momentum conservation in Maxwell-like form. The authors and their colleagues have found these Maxwell-like momentum tools useful for developing physical intuition into numericalrelativity simulations of compact binaries with spin.
\end{abstract}

DOI: $10.1103 /$ PhysRevD.80.124014

PACS numbers: 04.25.Nx, 04.20.Cv, 04.25.-g

\section{INTRODUCTION}

In 1961, Robert L. Forward [1] (building on earlier work of Einstein [2,3] and especially Thirring [4,5]) wrote the linearized, slow-motion approximation to general relativity in a form that closely resembles Maxwell's equations; and he displayed this formulation's great intuitive and computational power. In the half century since then, this Maxwell-like formulation and variants of it have been widely explored and used; see e.g. [6-13] and references therein.

In 1965-1969 S. Chandrasekhar [14,15] formulated the first post-Newtonian (weak-gravity, slow-motion) approximation to general relativity in a manner that has been widely used for astrophysical calculations during the subsequent 40 years. When linearized, this first postNewtonian (1PN) approximation can be (and often is) recast in Maxwell-like form.

In 1991, T. Damour, M. Soffel, and C. Xu (DSX [16]) extended this Maxwell-like 1PN formalism to include all 1PN nonlinearities (see also Sec. 13 of Jantzen, Carini, and Bini [17]). DSX did so as a tool in developing a general formalism for the celestial mechanics of bodies that have arbitrary internal structures and correspondingly have external gravitational fields characterized by two infinite sets of multipole moments. (For a generalization to scalartensor theories, see [18].) In 2004, Racine and Flanagan [19] generalized DSX to a system of compact bodies (e.g. black holes) that have arbitrarily strong internal gravity.

During the past 18 months, we and our colleagues have been exploring the flow of gravitational field momentum in numerical-relativity simulations of compact, spinning binaries $[20,21]$. In their inspiral phase, these binaries' mo- tions and precessions can be described by the 1PN approximation, ${ }^{1}$ and we have gained much insight into their dynamics by using the 1PN DSX Maxwell-like formalism, extended to include Maxwell-like momentum density, momentum flux, and momentum conservation. ${ }^{2}$

In this paper, we present that extension of DSX, ${ }^{3}$ though with two specializations: (i) we fix our coordinates (gauge) to be fully harmonic instead of maintaining the partial gauge invariance of DSX, and (ii) we discard all multipole moments of the binaries' bodies except their masses and their spin angular momenta, because for black holes and neutron stars, the influences of all other moments are numerically much smaller than $1.5 \mathrm{PN}$ order.

The DSX celestial-mechanics papers [16,23-25] are so long and complex that it is not easy to extract from them the bare essentials of the DSX Maxwell-like 1PN formalism. For the benefit of researchers who want those bare

\footnotetext{
${ }^{1}$ For black-hole and neutron-star binaries, the influences of spin that interest us are formally $1.5 \mathrm{PN}$, but because of the bodies' compactness (size of order Schwarzschild radius), they are numerically $1 \mathrm{PN}$.

${ }^{2} \mathrm{~A}$ referee has pointed out to us that some papers in the rich literature on the Maxwell-like formulation of linearized 1PN theory, e.g. [10], argue that the Maxwell analogy is physically useful only for stationary phenomena. Our spinning-binary application [20] of the momentum-generalized DSX formalism is a counterexample.

${ }^{3}$ When we carried out our analysis and wrote it up in the original version of this paper, we were unaware of the Maxwelllike formalism in DSX [16]; see our preprint at [22]. When we learned of DSX from Luc Blanchet, we used it to improve our Maxwell-like treatment of gravitational momentum (by replacing our definition for the gravitoelectric field with that of DSX) and we rewrote this paper to highlight the connection to DSX.
} 
essentials and want to see how they are related to more conventional approaches to 1PN theory, we summarize them before presenting our momentum extension, and we do so for a general stress-energy tensor, for a perfect fluid, and for a system of compact bodies described by their masses and spins.

This paper is organized as follows. In Sec. II we summarize the basic DSX equations for 1PN theory in Maxwell-like form. In Sec. III we specialize the DSX formalism to a perfect fluid and make contact with the conventional 1PN notation. In Sec. IV we extend DSX by deriving the (Landau-Lifshitz-based) density and flux of gravitational momentum in terms of the DSX gravitoelectric and gravitomagnetic fields and by writing down the law of momentum conservation in terms of them. (It is this that we have found so useful for gaining intuition into numerical-relativity simulations of inspiraling, spinning binaries [20,21].) In Sec. V we briefly discuss energy conservation. In Sec. VI, relying on Racine and Flanagan [19], we specialize to the vacuum in the near zone of a system made from compact bodies with arbitrarily strong internal gravity. Finally, in Sec. VII we summarize the DSX formalism and our extension of it both for a selfgravitating fluid and for a system of compact bodies.

Throughout this paper, we set $G=c=1$, Greek letters run from 0 to 3 (spacetime) and Latin from 1 to 3 (space), and we use the notation of field theory in flat space in a $3+1$ split, so spatial indices are placed up or down equivalently and repeated spatial indices are summed whether up or down or mixed. We use boldface italic characters to represent spatial vectors, e.g. $w$ is the boldface version of $w_{j}$.

\section{THE DSX MAXWELL-LIKE FORMULATION OF 1PN THEORY}

Damour, Soffel, and Xu (DSX [16]) express the 1PN metric in terms of two gravitational potentials, a scalar $w$ and a vector $w_{j}$ :

$$
\begin{aligned}
& g_{00}=-e^{-2 w}=-1+2 w-2 w^{2}+O\left(U_{N}^{3}\right), \\
& g_{0 i}=-4 w_{i}+O\left(U_{N}^{5 / 2}\right), \\
& g_{i j}=\delta_{i j} e^{2 w}=\delta_{i j}(1+2 w)+O\left(U_{N}^{2}\right)
\end{aligned}
$$

[DSX Eqs. (3.3)]. The Newtonian limit of $w$ is $U_{N}=$ (Newtonian gravitational potential), and $w_{i}$ is of order $U_{N}^{3 / 2}$ :

$$
w=U_{N}+O\left(U_{N}^{2}\right), \quad w_{i}=O\left(U_{N}^{3 / 2}\right) .
$$

The harmonic gauge condition implies that

$$
w_{, t}+w_{j, j}=0
$$

[DSX Eq. (3.17a)]; here and throughout commas denote partial derivatives. Using this gauge condition (which DSX do not impose), the 1PN Einstein field equations take the following remarkably simple form:

$$
\begin{aligned}
\nabla^{2} w-\ddot{w} & =-4 \pi\left(T^{00}+T^{j j}\right)+O\left(U_{N}^{3} / \mathcal{L}^{2}\right), \\
\nabla^{2} w_{i} & =-4 \pi T^{0 i}+O\left(U_{N}^{5 / 2} / \mathcal{L}^{2}\right)
\end{aligned}
$$

[DSX Eqs. (3.11)]. Here $T^{\alpha \beta}$ is the stress-energy tensor of the source (which we specialize below to a perfect fluid), $\nabla^{2}$ is the flat-space Laplacian (i.e. $\nabla^{2} w=w, j$ ) , repeated indices are summed, dots denote time derivatives (i.e. $\ddot{w}=$ $w_{, t t}$ ), and $\mathcal{L}$ is the length scale on which $w$ varies.

Following DSX, we introduce the 1PN gravitoelectric field $\boldsymbol{g}$ (denoted $\boldsymbol{e}$ or $\boldsymbol{E}$ by DSX, depending on the context) and gravitomagnetic field $\boldsymbol{H}$ (denoted $\boldsymbol{b}$ or $\boldsymbol{B}$ by DSX):

$$
\begin{gathered}
\boldsymbol{g}=\boldsymbol{\nabla} w+4 \dot{\boldsymbol{w}}+O\left(U_{N}^{3} / \mathcal{L}\right), \\
\boldsymbol{H}=-4 \boldsymbol{\nabla} \times \boldsymbol{w}+O\left(U_{N}^{5 / 2} / \mathcal{L}\right)
\end{gathered}
$$

[DSX Eqs. (3.21)].

The Einstein equations (2.4) and these definitions imply the following 1PN Maxwell-like equations for $\boldsymbol{g}$ and $\boldsymbol{H}$ :

$$
\begin{aligned}
\boldsymbol{\nabla} \cdot \boldsymbol{g} & =-4 \pi\left(T^{00}+T^{j j}\right)-3 \ddot{w}+O\left(g U_{N}^{2} / \mathcal{L}\right), \\
\nabla \times \boldsymbol{g} & =-\dot{\boldsymbol{H}}+O\left(g U_{N}^{2} / \mathcal{L}\right), \\
\boldsymbol{\nabla} \cdot \boldsymbol{H} & =0+O\left(H U_{N} / \mathcal{L}\right), \\
\boldsymbol{\nabla} \times \boldsymbol{H} & =-16 \pi T^{0 i} \boldsymbol{e}_{i}+4 \dot{\boldsymbol{g}}+O\left(H U_{N} / \mathcal{L}\right)
\end{aligned}
$$

[DSX Eqs. (3.22)]. Here $\boldsymbol{e}_{i}$ is the unit vector in the $i$ direction.

In terms of $\boldsymbol{g}$ and $\boldsymbol{H}$, the geodesic equation for a particle with ordinary velocity $\boldsymbol{v}=d \boldsymbol{x} / d t$ takes the following form [Eq. (7.17) of DSX, though in a less transparently "Lorentz-force"-like form there]:

$$
\begin{aligned}
\frac{d}{d t}\left[\left(1+3 U_{N}+\frac{1}{2} \boldsymbol{v}^{2}\right) \boldsymbol{v}\right]= & \left(1-U_{N}+\frac{3}{2} \boldsymbol{v}^{2}\right) \boldsymbol{g}+\boldsymbol{v} \times \boldsymbol{H} \\
& +O\left(g U_{N}^{2}\right) .
\end{aligned}
$$

Note that the spatial part of the particle's 4-momentum is $m \boldsymbol{u}=m\left(1+U_{N}+\frac{1}{2} \boldsymbol{v}^{2}\right) \boldsymbol{v}$ at 1PN order. This accounts for the coefficient $1+U_{N}+\frac{1}{2} \boldsymbol{v}^{2}$ on the left-hand side of Eq. (2.7). The remaining factor $2 U_{N}$ is related to the difference between physical lengths and times, and proper lengths and times. In the linearized, very-low-velocity approximation, this geodesic equation takes the "Lorentz-force" form $d \boldsymbol{v} / d t=\boldsymbol{g}+\boldsymbol{v} \times \boldsymbol{H}$, first deduced (so far as we know) in 1918 by Thirring [4], motivated by Einstein's 1913 [2] insights about similarities between electromagnetic theory and his not-yet-perfected general relativity theory.

The 1PN deviations of the geodesic equation (2.7) from the usual Lorentz-force form might make one wonder about the efficacy of the DSX definition of $\boldsymbol{g}$. That efficacy will show up most strongly when we explore the gravitational momentum density in Sec. IV below. 


\section{SPECIALIZATION TO A PERFECT FLUID}

We now depart from DSX by specializing our source to a perfect fluid and making contact with a set of 1PN gravitational potentials that are widely used. We pay special attention to connections with a paper by Pati and Will [26] because that paper will be our foundation, in Sec. IV, for computing the density and flux of gravitational field momentum.

We describe our perfect fluid in the following standard notation: $\rho_{o}=$ (density of rest mass), $\Pi=$ (internal energy per unit rest mass, i.e. specific internal energy, $P=$ (pressure), all as measured in the fluid's local rest frame; $v_{j} \equiv d x^{j} / d t=$ (fluid's coordinate velocity).

Following Blanchet and Damour [27], and subsequently Pati and Will (Eqs. (4.13), (4.3) of [26]), we introduce a post-Newtonian variant $U$ of the Newtonian potential, which is sourced by $T^{00}+T^{j j}: \nabla^{2} U=-4 \pi\left(T^{00}+T^{j j}\right)$. Accurate to 1PN order, the source is [see Eq. (4.4d) of Pati and Will]

$$
T^{00}+T^{j j}=\rho_{o}\left(1+\Pi+2 \boldsymbol{v}^{2}+2 U_{N}+3 P / \rho_{o}\right),
$$

where $U_{N}$ is the Newtonian limit of $U$

$$
U_{N}(\boldsymbol{x}, t)=\int \frac{\rho_{o}\left(\boldsymbol{x}^{\prime}, t\right)}{\left|\boldsymbol{x}-\boldsymbol{x}^{\prime}\right|} d^{3} x^{\prime} .
$$

Correspondingly, $U$ can be written as

$$
U=\int \frac{\rho_{o}\left(1+\Pi+2 \boldsymbol{v}^{2}+2 U_{N}+3 P / \rho_{o}\right)}{\left|\boldsymbol{x}-\boldsymbol{x}^{\prime}\right|} d^{3} x^{\prime} .
$$

Here and below the fluid variables and gravitational potentials in the integrand are functions of $\left(\boldsymbol{x}^{\prime}, t\right)$ as in Eq. (3.2a). In Eq. (3.2a) for $U_{N}, \rho_{o}$ can be replaced by any quantity that agrees with $\rho_{o}$ in the Newtonian limit, e.g. by the postNewtonian "conserved mass density" $\rho_{*}$ of Eq. (3.6b) below. We also introduce Chandrasekhar's postNewtonian scalar gravitational potential $\chi$ (Eq. (44) of [14]), which is sourced by $2 U_{N}, \nabla^{2} \chi=-2 U_{N}$ or equivalently

$$
\chi=-\int \rho_{o}\left|x-x^{\prime}\right| d^{3} x^{\prime}
$$

Pati and Will use the notation $-X$ for $\chi$ (Eqs. (4.14), (4.12a), and (4.3) of [26]).

It is straightforward to show that the 1PN solution to the wave equation (2.4a) for the DSX scalar potential $w$ is

$$
w=U-\frac{1}{2} \ddot{\chi}
$$

and the 1PN solution to the Laplace equation (2.4b) for the DSX vector potential $w_{j}$ is

$$
w_{j}=\int \frac{\rho_{o} v_{j}}{\left|\boldsymbol{x}-\boldsymbol{x}^{\prime}\right|} d^{3} x^{\prime}
$$

The fluid's evolution is governed by rest-mass conservation, momentum conservation, and energy conservation.

The 1PN version of rest-mass conservation takes the following form:

$$
\rho_{*, t}+\boldsymbol{\nabla} \cdot\left(\rho_{*} \boldsymbol{v}\right)=0,
$$

where

$$
\rho_{*}=\rho_{o} u^{0} \sqrt{-g}=\rho_{o}\left(1+\frac{1}{2} \boldsymbol{v}^{2}+3 U\right)
$$

(Eqs. (117) and (118) of Chandrasekhar [14]). Here $u^{0}$ is the time component of the fluid's 4-velocity and $g$ is the determinant of the covariant components of the metric.

We shall discuss momentum conservation and energy conservation in the next two sections.

We note in passing that Chandrasekhar and many other researchers write their 1PN spacetime metric in a different gauge from our harmonic one. The two gauges are related by a change of time coordinate

$$
t_{\mathrm{C}}=t_{\mathrm{H}}-\frac{1}{2} \dot{\chi},
$$

and correspondingly the metric components in the two gauges are related by

$$
g_{00}^{\mathrm{C}}=g_{00}^{\mathrm{H}}+\ddot{\chi}, \quad g_{0 j}^{\mathrm{C}}=g_{0 j}^{\mathrm{H}}+\frac{1}{2} \dot{\chi}, j .
$$

Here $\mathrm{C}$ refers to the Chandrasekhar gauge and $\mathrm{H}$ to our harmonic gauge. DSX write their equations in forms that are invariant under the gauge change (3.7).

\section{MOMENTUM DENSITY, FLUX, AND CONSERVATION}

We now turn to our extension of the DSX formalism to include a Maxwell-like formulation of gravitational momentum density, momentum flux, and momentum conservation. Following Chandrasekhar [15], Pati and Will [26] and others, we adopt the Landau-Lifshitz pseudotensor as our tool for formulating these concepts.

From Pati and Will's 2PN harmonic-gauge Eqs. (2.6, $4.4 \mathrm{~b}$, and $4.4 \mathrm{c}$ ) for the pseudotensor, one can deduce the following 1PN expressions for the gravitational momentum density and momentum flux (stress) in terms of the DSX gravitoelectric and gravitomagnetic fields $\boldsymbol{g}$ and $\boldsymbol{H}$ :

$$
\begin{aligned}
(-g) t_{\mathrm{LL}}^{0 j} \boldsymbol{e}_{j}= & -\frac{1}{4 \pi} \boldsymbol{g} \times \boldsymbol{H}+\frac{3}{4 \pi} \dot{U}_{N} \boldsymbol{g}, \\
(-g) t_{\mathrm{LL}}^{i j}= & \frac{1}{4 \pi}\left(g_{i} g_{j}-\frac{1}{2} \delta_{i j} g_{k} g_{k}\right) \\
& +\frac{1}{16 \pi}\left(H_{i} H_{j}-\frac{1}{2} \delta_{i j} H_{k} H_{k}\right)-\frac{3}{8 \pi} \dot{U}_{N}^{2} \delta_{i j} .
\end{aligned}
$$

Each equation is accurate up to corrections of order $U_{N}$ times the smallest term on the right side (2PN corrections).

For comparison, in flat spacetime the electromagnetic momentum density is $\frac{1}{4 \pi} \boldsymbol{E} \times \boldsymbol{B}$ and the momentum flux is $\frac{1}{4 \pi}\left(E_{i} E_{j}-\frac{1}{2} \delta_{i j} E_{k} E_{k}\right)+\frac{1}{4 \pi}\left(B_{i} B_{j}-\frac{1}{2} \delta_{i j} B_{k} B_{k}\right)$. Aside from a sign in Eq. (4.1a) and the two terms involving $\dot{U}_{N}$, the gravitational momentum flux and density (4.1) are identical to the electromagnetic ones with $\boldsymbol{E} \rightarrow \boldsymbol{g}$ and 
$\boldsymbol{B} \rightarrow \boldsymbol{H} / 2$. Therefore, by analogy with the electromagnetic case, there are gravitational tensions $|g|^{2} / 8 \pi$ and $|\boldsymbol{H}|^{2} / 32 \pi$ parallel to gravitoelectric and gravitomagnetic field lines, and gravitational pressures of these same magnitudes orthogonal to the field lines. This makes the gravitoelectric and gravitomagnetic fields $\boldsymbol{g}$ and $\boldsymbol{H}$ powerful tools for building up physical intuition about the distribution and flow of gravitational momentum. We use them for that in our studies of compact binaries [20,21], relying heavily on Eqs. (4.1).

Here are some hints for deducing Eqs. (4.1) from Pati and Will [26] (henceforth PW): (i) Show that the last two terms in (2.6) of PW are of $2 \mathrm{PN}$ order for $\{\alpha, \beta\}=\{0 j\}$ or $\{i j\}$ and so can be ignored, when $16 \pi(-g) t_{\mathrm{LL}}^{\alpha \beta}=\Lambda^{\alpha \beta}$. (ii) Show that our notation is related to that of PW by $\chi=$ $-X, U$ the same, $w=U-\frac{1}{2} \ddot{\chi}=\frac{1}{4}(N+B)-\frac{1}{8}(N+B)^{2}$ [for the last of these cf. PW (5.2) and (5.4a, c)], and at Newtonian order $U_{N}=\frac{1}{4} N$. (iii) In PW $(4.4 \mathrm{~b}, \mathrm{c})$ for $\Lambda^{\alpha \beta}$, keep only the Newtonian and 1PN terms: the first curly bracket in (4.4b) and first and second curly brackets in (4.4c). Rearrange those terms so they involve only $\boldsymbol{K}, N+$ $B$ and the Newtonian-order $N$, use the above translation of notation and use the definitions (2.5) of $\boldsymbol{g}$ and $\boldsymbol{H}$. Thereby, bring PW (4.4b and 4.4c) into the form (4.1).

In the Landau-Lifshitz formalism, the local law of 4momentum conservation $T_{; \mu}^{j \mu}=0$ takes the form

$$
\left[(-g)\left(T^{j \mu}+t_{\mathrm{LL}}^{j \mu}\right)\right]_{, \mu}=0
$$

(Eqs. (20.23a) and (20.19) of [28], or (100.8) of [29]). Here (as usual), commas denote partial derivatives, and semicolons denote covariant derivatives. This is the conservation law that we use in our studies of momentum flow in compact binaries [20,21].

When dealing with material bodies (e.g. in DSX) rather than with the vacuum outside compact bodies, an alternative Maxwell-like version of momentum conservation is useful. Specifically, using expressions (4.1) and $(-g)=$ $1+4 U_{N}$ [from Eq. (2.1) with $w=U_{N}$ at leading order], and using the field equations (2.6) for $\boldsymbol{g}$ and $\boldsymbol{H}$, the conservation law (4.2) can be rewritten in the following simple Lorentz-force-like form (Eq. (4.3) of Damour, Soffel, and Xu's paper II [23])

$$
\begin{gathered}
{\left[\left(1+4 U_{N}\right) T^{i 0}\right]_{, t}+\left[\left(1+4 U_{N}\right) T^{i j}\right]_{, j}} \\
\quad=\left(T^{00}+T^{j j}\right) g_{i}+\epsilon_{i j k} T^{0 j} H_{k} .
\end{gathered}
$$

Here the Levi-Civita tensor $\epsilon_{i j k}$ produces a cross product of the momentum density with the gravitomagnetic field. For comparison, in flat spacetime, the momentum conservation law for a charged medium interacting with electric and magnetic fields $E_{i}$ and $B_{i}$ has the form $T^{i 0}{ }_{, t}+T^{i j}{ }_{, j}=$ $\rho_{e} E_{i}+\epsilon_{i j k} J_{j} B_{k}$, where $\rho_{e}$ is the charge density and $J_{j}$ the charge flux (current density). The right-hand side of Eq. (4.3) (the gravitational force density) is identical to that in the electromagnetic case, with $\rho_{e} \rightarrow\left(T^{00}+T^{j j}\right)$,
$J_{j} \rightarrow T^{0 j}, \boldsymbol{E} \rightarrow \boldsymbol{g}$, and $\boldsymbol{B} \rightarrow \boldsymbol{H}$. Again, this makes $\boldsymbol{g}$ and $\boldsymbol{H}$ powerful foundations for gravitational intuition.

For a perfect fluid, the components of the 1PN stressenergy tensor, which appear in the momentum conservation law (4.3), are (Eqs. (20) of Chandrasekhar [14])

$$
\begin{aligned}
T^{00}= & \rho_{o}\left(1+\Pi+\boldsymbol{v}^{2}+2 U_{N}\right), \\
T^{i 0}= & \rho_{o}\left(1+\Pi+\boldsymbol{v}^{2}+2 U_{N}+P / \rho_{o}\right) v_{j}, \\
T^{i j}= & \rho_{o}\left(1+\Pi+\boldsymbol{v}^{2}+2 U_{N}+P / \rho_{o}\right) v_{i} v_{j} \\
& +P\left(1-2 U_{N}\right) \delta_{i j}, \\
T^{00}+T^{j j}= & \rho\left(1+\Pi+2 \boldsymbol{v}^{2}+2 U_{N}+3 P / \rho_{o}\right) .
\end{aligned}
$$

\section{ENERGY CONSERVATION}

For a perfect fluid, the exact (not just 1PN) law of energy conservation, when combined with mass conservation and momentum conservation, reduces to the first law of thermodynamics $d \Pi / d t=-P d\left(1 / \rho_{o}\right) / d t$; so whenever one needs to invoke energy conservation, the first law is the simplest way to do so. For this reason, and because deriving the explicit form of 1PN energy conservation $[(-g) \times$ $\left.\left(T^{0 \mu}+t_{\mathrm{LL}}^{0 \mu}\right)\right]_{, \mu}=0$ is a very complex and delicate task (cf. Sec. VI of [30]), we shall not write it down here.

However, we do write down the Newtonian law of energy conservation in harmonic gauge, since we will occasionally need it in our future papers. Chandrasekhar calculated $(-g)\left(T^{0 \mu}+t_{\mathrm{LL}}^{0 \mu}\right)$ in [15]; his Eqs. (48) and (57) are the time-time and time-space components, respectively. When one writes the expressions in terms of the "conserved rest-mass density" $\rho_{*}$ [Eq. (3.6b)] and in our Maxwell-like form, Newtonian conservation of energy states that

$$
\begin{aligned}
{\left[\rho_{*}\left(1+\Pi+\frac{1}{2} \boldsymbol{v}^{2}+3 U_{N}\right)-\frac{7}{8 \pi} \boldsymbol{g} \cdot \boldsymbol{g}\right]_{, t} } \\
+\boldsymbol{\nabla} \cdot\left[\rho_{*} \boldsymbol{v}\left(1+\Pi+\frac{P}{\rho}+\frac{1}{2} \boldsymbol{v}^{2}+3 U_{N}\right)+\frac{3}{4 \pi} \dot{U}_{N} \boldsymbol{g}\right. \\
\left.\quad-\frac{1}{4 \pi} \boldsymbol{g} \times \boldsymbol{H}\right]=0
\end{aligned}
$$

While this equation is perfectly correct, it expresses Newtonian energy conservation in terms of the postNewtonian gravitomagnetic field $\boldsymbol{H}$. It is possible to rewrite the $\boldsymbol{H}$-dependent term using the relationship, $\nabla \cdot[-1 /(4 \pi)(\boldsymbol{g} \times \boldsymbol{H})]=\nabla \cdot\left[-4 U_{N} T^{0 j} \boldsymbol{e}_{j}+(1 / \pi) U_{N} \dot{\boldsymbol{g}}\right]$, which is accurate up to corrections of order $\boldsymbol{g} \cdot \dot{\boldsymbol{H}}$. This relationship can be found by applying Eq. (2.6b) once and (2.6d) twice, in combination with elementary vectorcalculus identities. The statement of Newtonian energy conservation then depends only upon the Newtonian potential and its gradient and time derivative: 


$$
\begin{array}{r}
{\left[\rho_{*}\left(1+\Pi+\frac{1}{2} \boldsymbol{v}^{2}+3 U_{N}\right)-\frac{7}{8 \pi} \boldsymbol{g} \cdot \boldsymbol{g}\right]_{, t}} \\
+\boldsymbol{\nabla} \cdot\left[\rho_{*} \boldsymbol{v}\left(1+\Pi+\frac{P}{\rho}+\frac{1}{2} \boldsymbol{v}^{2}-U_{N}\right)+\frac{3}{4 \pi} \dot{U}_{N} \boldsymbol{g}\right. \\
\left.+\frac{1}{\pi} U_{N} \dot{\boldsymbol{g}}\right]=0 .
\end{array}
$$

Notice that going from Eq. (5.1) to Eq. (5.2) involves adding a divergence-free piece to the energy flux, so it entails changing how the energy flux is localized-a change that strictly speaking takes the energy flux out of harmonic gauge.

If the coefficients of the gravitational terms in Eq. (5.2) look unfamiliar, it is because even at Newtonian order, the density and flux of gravitational energy are gaugedependent. In some other gauge, they will be different; see Box 12.3 of [31].

\section{GRAVITATIONAL POTENTIALS IN THE VACUUM OF A SYSTEM OF COMPACT, SPINNING BODIES}

For a system of compact, spinning bodies (neutron stars or black holes), the gravitational potentials $U_{N}, U, w_{i}$, and $\chi$ in the vacuum between the bodies take the following forms (in a slightly different harmonic gauge than the one used in Sec. III for fluids):

$$
\begin{aligned}
U_{N} & =\sum_{A} \frac{M_{A}}{r_{A}} \\
U & =\sum_{A} \frac{M_{A}}{r_{A}}\left(1+\frac{3}{2} \boldsymbol{v}_{A}^{2}-\sum_{B \neq A} \frac{M_{B}}{r_{A B}}\right)+2 \sum_{A} \frac{\epsilon_{i j k} v_{A}^{i} S_{A}^{j} n_{A}^{k}}{r_{A}^{2}} \\
\chi & =-\sum_{A} M_{A} r_{A} \\
w & =U-\frac{1}{2} \ddot{\chi} \\
w_{i} & =\sum_{A} \frac{M_{A} v_{A}^{i}}{r_{A}}+\frac{1}{2} \sum_{A} \frac{\epsilon_{i j k} S_{A}^{j} n_{A}^{k}}{r_{A}{ }^{2}}
\end{aligned}
$$

Here the notation is that of Sec. IV of Thorne and Hartle [32]: the sum is over the compact bodies labeled by capital Latin letters $A, B ; M_{A}, S_{A}^{j}$ and $v_{A}^{j}$ are the mass, spin angular momentum and coordinate velocity of body $A ; r_{A}$ is the coordinate distance from the field point to the center of mass of body $A ; r_{A B}$ is the coordinate distance between the centers of mass of bodies $A$ and $B ; n_{A}^{j}$ is the unit vector pointing from the center of mass of body $A$ to the field point; and $\epsilon_{i j k}$ is the Levi-Civita tensor.
Equations (6.1) for the potentials can be deduced by comparing our 1PN spacetime metric coefficients [Eqs. (2.1) and (3.4)] with those in Eqs. (2.4), (5.11) and (5.14) of Racine and Flanagan [19] or in Eqs. (4.4) of Thorne and Hartle [32]. ${ }^{4}$

\section{CONCLUSION}

In our Maxwell-like formulation of the 1PN approximation to general relativity for fluid bodies, the evolution of the fluid and gravitational fields is governed by: (i) the law of momentum conservation (4.3) and (4.4) (which can be thought of as evolving the fluid velocity $v_{j}$ ); (ii) the law of mass conservation (3.6) (which can be thought of as evolving the mass density $\left.\rho_{o}\right)$; (iii) the equation of state $P\left(\rho_{o}\right)$ and first law of thermodynamics $d \Pi=-P d\left(1 / \rho_{o}\right)$ (which determine $P$ and $\Pi$ once $\rho_{o}$ is known); Eqs. (3.2), (3.3), and (3.5) for the gravitational potentials $U, \chi$, and $w_{j}$; and Eqs. (2.5) or (2.6) for the gravitoelectric and gravitomagnetic fields $\boldsymbol{g}, \boldsymbol{H}$.

When specialized to a system of compact bodies, e.g. a binary made of black holes or neutron stars, the system is governed by: (i) $1 \mathrm{PN}$ equations of motion and precession for the binary (not given in this paper; see e.g. Eqs. (4.10), (4.11), and (4.14) of [32]); (ii) momentum flow within the binary as described by the Landau-Lifshitz pseudotensor (4.1) and its conservation law (4.2), in which the gravitoelectric and gravitomagnetic fields are expressed as sums over the bodies via Eqs. (6.1) and (2.5); and (iii) other tools developed by Landau and Lifshitz (Sec. 100 of [29]). We are finding this formalism powerful in gaining insight into compact binaries [20,21].

\section{ACKNOWLEDGMENTS}

For helpful discussions we thank Yanbei Chen and Drew Keppel. We also thank Luc Blanchet for bringing references $[16,27]$ to our attention, and anonymous referees for helpful critiques and suggestions. This research was supported in part by NSF Grant Nos.PHY-0601459 and PHY0653653.

\footnotetext{
${ }^{4}$ Racine and Flanagan specialize DSX to a system of compact bodies with a complete set of nonzero multipole moments. We neglect all moments except the bodies' masses and spins (see fifth paragraph of Sec. I). Our notation is related to that of Racine and Flanagan by $U_{N}=-\Phi, w=U-\frac{1}{2} \ddot{\chi}=-(\Phi+\psi), w_{i}=$ $-\frac{1}{4} \zeta_{i}$. The Racine-Flanagan derivation of Eqs. (6.1) avoids considering the internal structures of the bodies and it therefore is directly valid for black holes. The Thorne-Hartle derivation relies on the pioneering analysis of Einstein, Infeld, and Hoffman [33] which uses a fluid description of the bodies' interiors. Thorne and Hartle extend Eqs. (6.1) to black holes by the equivalent of Damour's "effacement" considerations, Sec. 6.4 of [34].
} 
[1] R. L. Forward, Proc. IRE 49, 892 (1961).

[2] A. Einstein, Phys. Z. 14, 1261 (1913).

[3] A. Einstein, Four Lectures on the Theory of Relativity, English translation by Edwin Plimpton Adams (Princeton University, Princeton, NJ, 1922); in The Collected Papers of Albert Einstein (Princeton University, Princeton, NJ, 2002), Vol. 7, see p. 100-103.

[4] H. Thirring, Phys. Z. 19, 204 (1918).

[5] H. Thirring, Phys. Z. 19, 33 (1918); H. Thirring and J. Lense, Phys. Z. 19, 156 (1918); English translations in B. Mashhoon, F. W. Hehl, and D.S. Theiss, Gen. Relativ. Gravit. 16, 711 (1984).

[6] V. B. Braginsky, C. M. Caves, and K. S. Thorne, Phys. Rev. D 15, 2047 (1977).

[7] R. M. Wald, General Relativity (University of Chicago, Chicago, 1984), Sec. 4.4a.

[8] B. Mashhoon, Phys. Lett. A 173, 347 (1993).

[9] B. Mashhoon, F. Gronwald, and H. I. M. Lichtenegger, Lect. Notes Phys. 562, 83 (2001).

[10] S. J. Clark and R. W. Tucker, Classical Quantum Gravity 17, 4125 (2000).

[11] B. Mashhoon, Classical Quantum Gravity 25, 085014 (2008).

[12] E. Goulart and F. T. Falciano, Int. J. Mod. Phys. A 24, 4589 (2009).

[13] L.F. O. Costa and C.A. R. Herdeiro, Phys. Rev. D 78, 024021 (2008).

[14] S. Chandrasekhar, Astrophys. J. 142, 1488 (1965).

[15] S. Chandrasekhar, Astrophys. J. 158, 45 (1969).

[16] T. Damour, M. Soffel, and C. Xu, Phys. Rev. D 43, 3273 (1991).

[17] R. T. Jantzen, P. Carini, and D. Bini, Ann. Phys. (N.Y.) 215, 1 (1992).

[18] S. Kopeikin and I. Vlasov, Phys. Rep. 400, 209 (2004).

[19] E. Racine and E. E. Flanagan, Phys. Rev. D 71, 044010 (2005).
[20] D. G. Keppel, D. A. Nichols, Y. Chen, and K. S. Thorne, following Article, Phys. Rev. D 80, 124015 (2009).

[21] G. Lovelace, Y. Chen, M. Cohen, J. D. Kaplan, D. Keppel, K. D. Matthews, D. A. Nichols, M. A. Scheel, and U. Sperhake, arXiv:0907.0869 [Phys. Rev. D (to be published)].

[22] J.D. Kaplan, D. A. Nichols, K. S. Thorne, arXiv: 0808.2510v1.

[23] T. Damour, M. Soffel, and C. Xu, Phys. Rev. D 45, 1017 (1992).

[24] T. Damour, M. Soffel, and C. Xu, Phys. Rev. D 47, 3124 (1993).

[25] T. Damour, M. Soffel, and C. Xu, Phys. Rev. D 49, 618 (1994).

[26] M.E. Pati and C.M. Will, Phys. Rev. D 62, 124015 (2000).

[27] L. Blanchet, T. Damour, Ann. Inst. Henri Poincaré 50, 337 (1989).

[28] C. W. Misner, K. S. Thorne, and J. A. Wheeler, Gravitation (Freeman, San Francisco, 1973).

[29] L. D. Landau and E. M. Lifshitz, Classical Theory of Fields (Addison-Wesley, Redding, MA, 1962).

[30] S. Chandrasekhar and Y. Nutku, Astrophys. J. 158, 55 (1969).

[31] R.D. Blandford and K.S. Thorne, Applications of Classical Physics http://www.pma.caltech.edu/Courses/ ph136/yr2008/.

[32] K. S. Thorne and J. B. Hartle, Phys. Rev. D 31, 1815 (1985).

[33] A. Einstein, L. Infeld, and B. Hoffman, Ann. Math. 39, 65 (1938).

[34] T. Damour, in Three Hundred Years of Gravitation, edited by S. W. Hawking and W. Israel (Cambridge University Press, Cambridge, England, 1987). 